REGARDS

SUR L'ECONOMIE ALLEMANDE

BULLETIN ECONOMIQUE DU CRAC

\section{Regards sur l'économie allemande}

Bulletin économique du CIRAC

$81 \mid 2007$

Varia

\title{
Innovation :l'industrie allemande leader en Europe
}

\section{Solène Hazouard}

\section{CpenEdition}

\section{Journals}

Édition électronique

URL : http://journals.openedition.org/rea/675

DOI : $10.4000 /$ rea. 675

ISBN : 978-2-8218-0858-4

ISSN : 1965-0787

Éditeur

CIRAC

Édition imprimée

Date de publication : 1 mai 2007

Pagination : 36-38

ISSN : 1156-8992

Référence électronique

Solène Hazouard, «Innovation :l'industrie allemande leader en Europe », Regards sur l'économie allemande [En ligne], 81 | mai 2007, document 2, mis en ligne le 01 mai 2009, consulté le 15 septembre 2020. URL : http://journals.openedition.org/rea/675

Ce document a été généré automatiquement le 15 septembre 2020

(c) CIRAC 


\title{
Innovation :l'industrie allemande leader en Europe
}

\author{
Solène Hazouard
}

1 Les entreprises industrielles allemandes sont les plus innovantes d'Europe et devancent par ailleurs la Corée du Sud et le Japon. C'est ce que démontre la dernière enquête sur l'innovation Innovationsverhalten der deutschen Wirtschaft, Indikatorenbericht zur Innovationserhebung 2006, menée au printemps dernier auprès de quelque 20000 entreprises et présentée le 26 février. Depuis 1993, les résultats de ce baromètre sont publiés chaque année par l'Institut ZEW de Mannheim, en partenariat avec l'Institut Fraunhofer de recherche sur les systèmes et l'innovation (ISI, Karlsruhe) et l'Institut des sciences sociales appliquées (Infas, Bonn) pour le compte du Ministère fédéral de la recherche.

\section{Le taux d'entreprises innovantes baisse, mais l'intensité de l'innovation s'accroît}

2 Malgré de très bons résultats à l'échelle européenne, le rythme de l'innovation a connu un léger ralentissement en Allemagne. Le taux d'entreprises innovantes est tombé de $47 \%$ à $45 \%$ entre 2004 et 2005 (le taux de 2006 devrait cependant retrouver le niveau de 2004). La croissance du budget de l'innovation en valeur nominale a légèrement fléchi elle aussi. Certes, les dépenses en innovation avaient connu une hausse de $5 \%$ entre 2004 et 2005 pour atteindre 106,8 milliards $€$; mais avec 108,1 milliards $€$, l'année 2006 s'est traduite par une moindre performance $(+1 \%$ seulement). Ce montant devrait très peu varier en 2007: le ZEW prévoit un volume de dépenses de 107,7 milliards $€$. Toutefois, estime l'institut, grâce à l'évolution conjoncturelle étonnamment favorable au deuxième semestre de 2006, une hausse plus nette du budget de l'innovation n'est pas à exclure.

3 Malgré ces signes de stagnation apparente, l'intensité de l'innovation ne cesse pourtant de s'accroître : en 2005, l'augmentation des dépenses d'innovation avait devancé d'un point celle du chiffre d'affaires global des entreprises $(+4 \%)$. Certes, $71 \%$ du budget de 
l'innovation revient aux grandes structures, mais, dans la mesure où les PME comptent pour plus de $99 \%$ des entreprises outre-Rhin, c'est bien le Mittelstand qui détermine le taux d'entreprises innovantes.

\section{Priorité à l'innovation process}

4 Si 47,5\% des entreprises industrielles se sont consacrées en 2005 à l'innovation produit, une part croissante d'entreprises tous secteurs confondus tend à accorder la priorité aux innovations process avec pour objectif une réduction des coûts. Pas moins de $37 \%$ des entreprises du secteur manufacturier $(+1,5 \%$ par rapport à 2004$)$ et de $36 \%$ des entreprises de services intensifs en savoir $(+8 \%)$ ont joué la carte de la nouveauté ou de l'amélioration process. Dans un environnement globalisé, il semble que cette stratégie l'emporte sur le choix du lancement de produits totalement nouveaux en période de demande modérée et de concurrence internationale accrue.

Mais les innovations process font aussi la part belle à l'amélioration de la qualité. Ainsi, elles ont permis à $28 \%$ des entreprises de services intensifs en savoir d'accroître la qualité de leurs prestations, tandis que $19 \%$ d'entre elles seulement les mettaient en œuvre pour réduire leurs coûts. Dans le secteur manufacturier, la quête de compétitivité occupe toujours une place déterminante: si $25 \%$ des entreprises innovent pour réduire leurs coûts, $27 \%$ le font pour développer la qualité de leurs produits, répondant en cela à une demande domestique et mondiale de plus en plus exigeante. Les autres entreprises de services comptent tout autant sur un perfectionnement de la qualité que sur la baisse des coûts (respectivement 12,5\%) afin de rester compétitives dans un contexte concurrentiel accru soit par l'évolution des activités (transports, intérim) soit par la libéralisation des marchés (services postaux). Dans l'ensemble, de plus en plus d'entreprises conjuguent aujourd'hui ces deux facteurs (coût et qualité), ce qui reflète la poursuite des efforts de rationalisation et la quête permanente d'efficience des entreprises allemandes.

Intensité de l'innovation dans les pays européens en 2004*

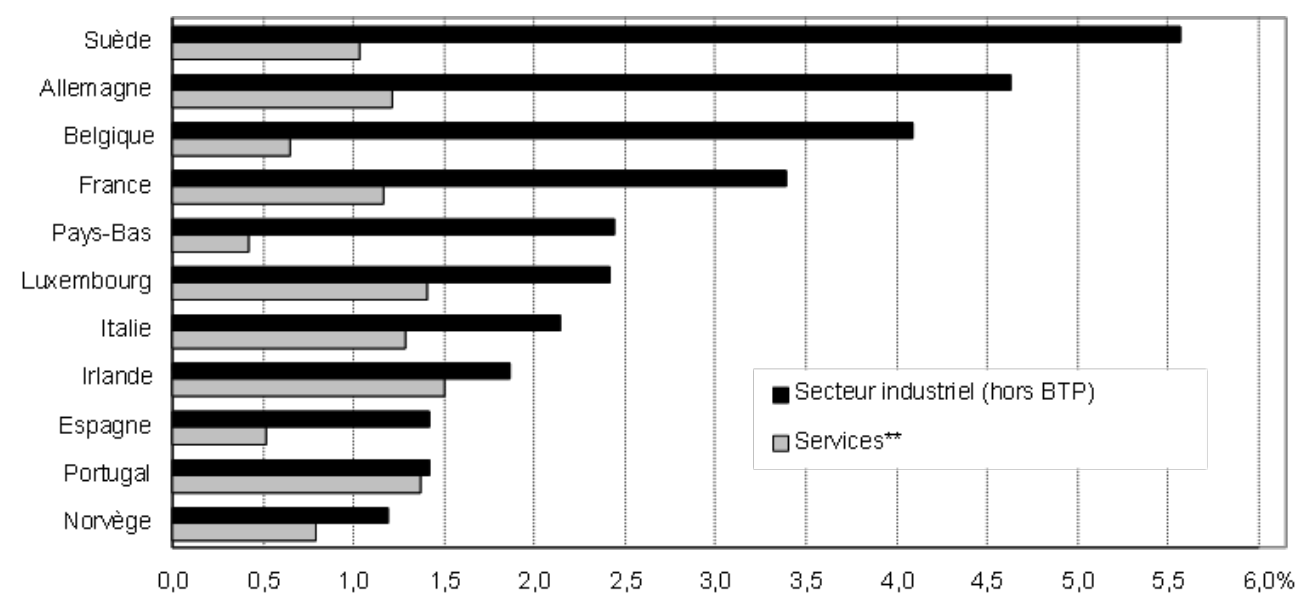

Source : Eurostat, $4^{e}$ enquête communautaire sur l'innovation CIS4 (Community Innovation Survey 4) Calculs du ZEW. Pas de données pour le Danemark, la Finlande et la Grande-Bretagne. *) Intensité de l'innovation : ensemble des dépenses d'innovation en \% du CA de toutes les entreprises de plus de 10 salariés. ${ }^{\star *}$ ) Commerce de gros, transport, télécommunications / TIC / bureautique, informatique, bureaux d'études, laboratoires techniques. 


\section{Intensité de l'innovation dans l'industrie : la RFA $2^{\mathrm{e}}$ dans I'UE}

6 Dans la logique de benchmarking classique outre-Rhin, le ZEW replace ces données nationales dans le contexte de la $4^{\mathrm{e}}$ enquête communautaire sur l'innovation (CIS4) publiée en 2007 par Eurostat, et dont les données se réfèrent à l'année 2004. Il apparaît que l'Allemagne, grâce notamment au dynamisme de ses PME, se trouve en tête en ce qui concerne le taux d'entreprises industrielles innovantes en 2004 (65\%), ainsi que la part du CA consacrée par le secteur manufacturier aux nouveautés produits $(24 \%)$. Toujours dans ce secteur, elle occupe le second rang juste derrière la Suède pour l'intensité de l'innovation. Mais dans les services, la vigilance reste de mise : certes en deuxième position derrière le Luxembourg pour la part d'entreprises innovantes, l'Allemagne n'est qu'au $5^{\mathrm{e}}$ rang pour l'innovation produit rapportée au CA comme pour l'intensité de l'innovation.

7 Si l'Allemagne est en tête en termes d'innovation industrielle, il n'est pas question pour autant pour elle de se reposer sur ses lauriers : l'évolution de certains indicateurs et le résultat légèrement plus mitigé des entreprises de services démontrent que les efforts doivent être poursuivis, voire s'intensifier. Ce n'est qu'à cette condition que la RFA pourra conserver sa position de leader européen, conclut le ZEW.

INDEX

Mots-clés : entreprise, Europe, industrie, innovation, UE 